\title{
Electron Microscopy of Human Endometrium during Window of Implantation
}

\author{
Patki SM*, Patki SS, Patil RS, Patki US, Patil PS, Sharma RK, Walawalkar S and Shah N
}

Obstetrics and Gynecology Department, Patki Research Foundation and Hospital Kolhapur, Maharashtra, India

"Corresponding author: Patki SM, Obstetrics and Gynecology Department, Patki Research Foundation and Hospital Kolhapur, Maharashtra, India, Tel: +91-9823388858; E-mail: satish.patki@rediffmail.com

Received date: July 08, 2018; Accepted date: August 08, 2018; Published date: August 14, 2018

Copyright: $\odot 2018$ Patki SM, et al. This is an open-access article distributed under the terms of the Creative Commons Attribution License, which permits unrestricted use, distribution, and reproduction in any medium, provided the original author and source are credited.

\begin{abstract}
Background: The implantation rate in assisted reproductive technology (ART) remains 25 to 35 percent inspite of marked improvement in the technology. Endometrium is receptive for the process of implantation of the blastocyst only for a span of 3 to 4 days of window of implantation (WOI). During this WOI, certain morphological changes take place in the luminal endometrium. Present study is an attempt to study these ultra-structural changes using scanning electron microscopy (SEM) during hormone replacement cycles (HRT) as the implantation rates are higher in such cycles than in stimulated cycles.
\end{abstract}

Material and Methods: Forty female infertile patients were given the hormone replacement regimen. $6 \mathrm{mg}$ Estradiol valerate was given per day from day 2 of menstrual cycle for 8days. Daily progesterone was supplemented from day 9 by intra muscular route in a dose of $100 \mathrm{mg}$ for 7 days. Sequential endometrial biopsies were performed on 2nd, 5th and 7th day of progesterone. The endometrial (SEM) tissues were subjected for scanning electron microscopy for studying the ultrastructural changes in the luminal endometrium.

Results: SEM showed the changes in all the three components of the surface endometrium. The surface epithetlium showed appearance pinopodes all along the surface on 2 nd day of progesterone. The pinopodes were found to be fully developed on 5th day and found to be regressed on 7 th day of progesterone. The endometrial glands were observed to be maximally developed in number and diameter on 5th day of progesterone. The phenomenon of angiogenesis was also maximally expressed on 5th day of progesterone.

Conclusion: Endometrial receptivity is maximally expressed on 5th day of progesterone administration in estrogenic primed patients. Documentation of these changes in one cycle prior to the treatment cycle will help for personalized embryo transfer in oocyte/embryo donation and frozen embryo transfer cycle, to get higher pregnancy rates.

Keywords: Endometrial receptivity; Window of implantation; Pinopodes; Scanning electronMicroscopy; Blastocyst

\section{Introduction}

In spite of marked improvement in assisted reproductive technology (ART), the implantation rate is 25 to 35 percent as the endometrial receptivity still remains a challenge [1]. Endometrium is receptive for the process of implantation of the blastocyst only for a span of 3 to 4 days, which is called as window of implantation (WOI) [2]. The window of endometrial receptivity is restricted to day 16 to 22 of 28 days normal cycle. During this WOI, anatomical, morphological and molecular changes take place in the endometrium leading ultimately to enable the blastocyst to attach \& finally invade the endometrial tissue. In ovarian stimulated cycles of ART, the levels of estradiol are higher (supraphysiological). Additionally, there are higher chances of premature LH (Leuteinising Hormone) surge, leading to raised level of progesterone prior to ovulation. These two hormonal events cause the appearance of early secretary changes in the endometrium and make it distorted for the process of implantation. In such cases the window of implantation is preponed and hence even if the good quality embryos are transferred the implantation rates are poor.
On the other hand in hormone replacement cycles the ovulation is suppressed and the sequential regimen of estradiol valertae for 8 to 10 days followed by addition of progesterone is administered. The endometrium in such cycle is developed in a synchronized way and the window of implantation is maintained in an ordered fashion between day 3 to day 5 of progesterone administration. Frozen embryos are transferred after throwing in this particular window. Similarly, in cases of oocyte donation and embryo donation, the procedure of embryo transfer is planned in this peculiar window. Interestingly, the implantation rates are higher in such hormone replacement cycles. Paulson et al. [3] and Edwards et al. [4] have shown that the clinical pregnancy rate is higher in hormone replacement therapy (HRT) cycles than in stimulated cycles, probably due to higher endometrial receptivity in HRT cycles. With this background, in the present study we have focused on the scanning electron microscopic study of the endometrium during day 2 to day 7 of progesterone administration of HRT cycle.

Earlier studies have documented the appearance of smooth, balloon like projections arising from the apical surface of the luminal epithelium of the endometrium during WOI observed by scanning electron microscopy (SEM). However, no study has been dedicatedly undertaken to study the luminal endometrium in HRT cycles. Present 
Citation: Patki SM, Patki SS, Patil RS, Patki US, Patil PS, et al. (2018) Electron Microscopy of Human Endometrium during Window of

Implantation. J Cytol Histol 9: 516. doi:10.4172/2157-7099.1000516

Page 2 of 5

study is the first of its kind study to evaluate the changes in all the components of luminal surface of the endometrium during WOI in HRT cycles. The understanding of these changes can be useful for the procedures of personalized embryo transfer for higher pregnancy rate in ART cycles.

\section{Materials and Methods}

The study was approved by the local committee for Ethics of Scientific Research. The duration of the study was from April 2013 to March 2015. Forty infertile patients undergoing In Vitro Fertilization (IVF) treatment by oocyte or embryo donation method were included for the study. Informed consent was obtained from the patients. The patients having systemic disorders like diabetes, hypertension and other infective diseases were excluded. The patients having pelvic inflammatory disease, fibroids endometriosis and other pelvic pathology were also excluded. Informed consent was obtained.

One cycle prior to the actual treatment cycle of embryo transfer was selected for the study. The patients were given orally estradiol valerate in the dose of $2 \mathrm{mg}$ thrice a day, starting from day 3 of the menstrual cycle. Transvaginal sonography (TVS) was performed, from day 9. Once the endometrium was $8 \mathrm{~mm}$ thick with a triple layer appearance on TVS, progesterone was added in dose of $100 \mathrm{mg}$ by intramuscular injection route daily for 7 days. The endometrial biopsies were sequentially taken by aseptic techniques on day 2,5 and 7 of progesterone administration. The endometrial tissue was washed with phosphate buffer saline several times to get rid of red blood cells. The bits of the washed endometrium were stored in the tube containing $1 \%$ glutaraldehyde solution at 4 degree Celsius till examination.

At the time of examination, the tissue was fixed with $4 \%$ glutaraldehyde. The process of dehydration of the tissue was done using serial dilutions of acetone starting from $10 \%$ to $100 \%$. The moisture was taken out by blooming air with rubber teat. The specimen was mounted on the stage of electron microscope by double sided carbon tape and the instrument was started to create a vacuum inside. The scanning electron microscope used for the study was of FE1 Quanta 200 SEM make which is a versatile, high performance, low vacuum instrument with a tungsten electron source with three imaging modes. The magnifications used were from $100 \mathrm{X}$ to $4000 \mathrm{X}$.

\section{Results}

The three important components of the surface luminal epithelium viz. surface epithelium, glands and vessels were studied. The stromal cells were at a deeper layer and hence could be not picked up in many cases. The surface epithelium was showing balloon like projections arising from the apical surface, called pinopodes. The biopsy on 2nd day of progesterone revealed very small pinopodes called as developing pinopodes (DP), that on 5th day of progesterone revealed fully developed pinopodes (FDP) while that on 7 th day revealed regressing pinopodes (RP) (Figures 1-3), the glands also showed a trend of increased number as well as diameter (Figures 4 and 5) when followed from day 2 to day 5 of progesterone, after which they became less prominent and regressed (Figure 6) Interestingly, the glands on day 6, were studded with pinopodes on the surface as well as throughout their depth.

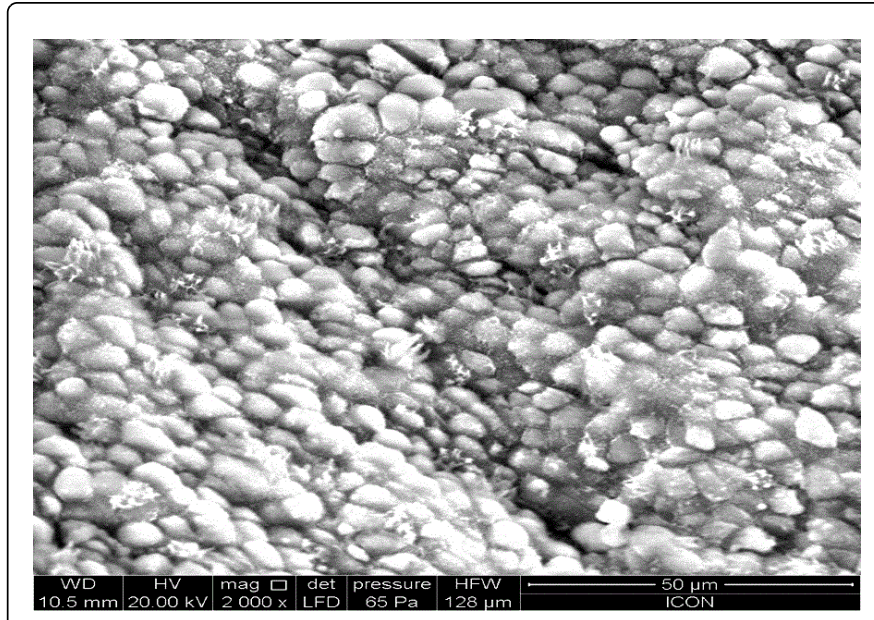

Figure 1: Developing pinopodes 2000X.

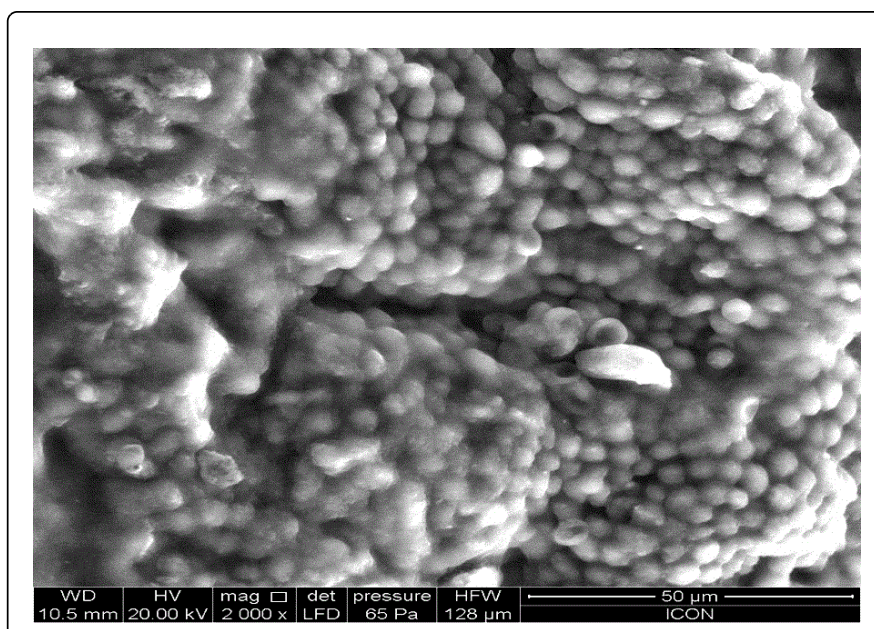

Figure 2: Developed pinopodes 2000X.

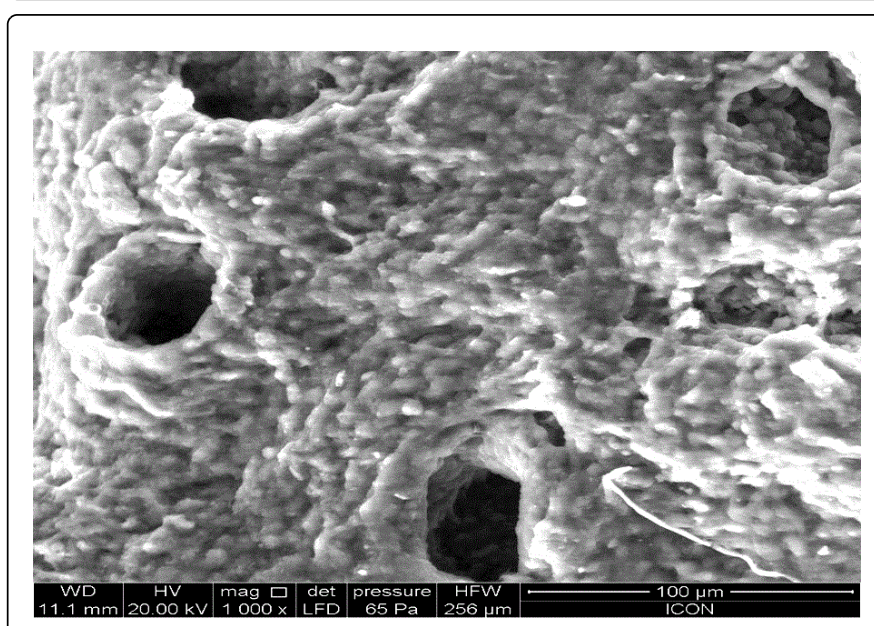

Figure 3: Developed glands 1000X. 
Citation: Patki SM, Patki SS, Patil RS, Patki US, Patil PS, et al. (2018) Electron Microscopy of Human Endometrium during Window of Implantation. J Cytol Histol 9: 516. doi:10.4172/2157-7099.1000516

Page 3 of 5

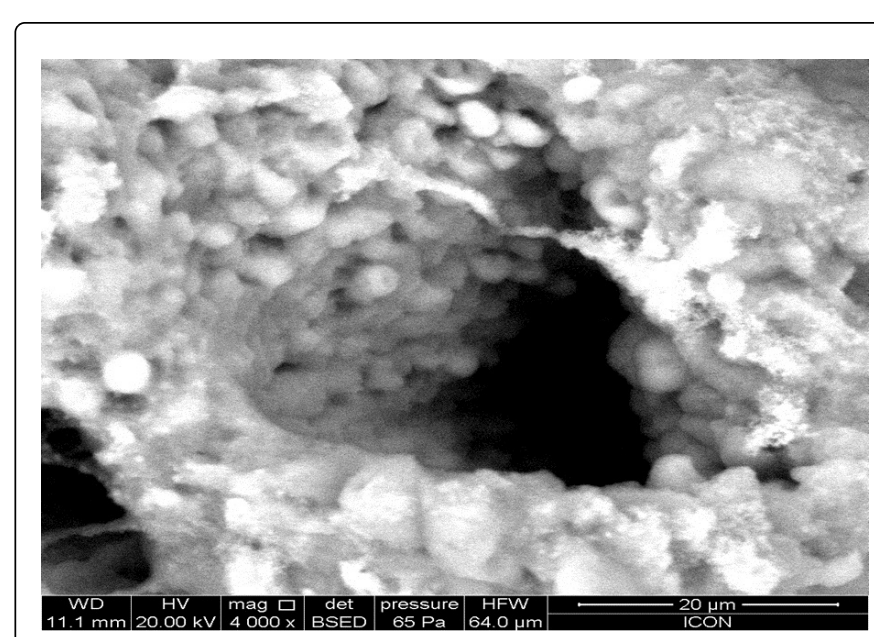

Figure 4: Developed gland 4000X.

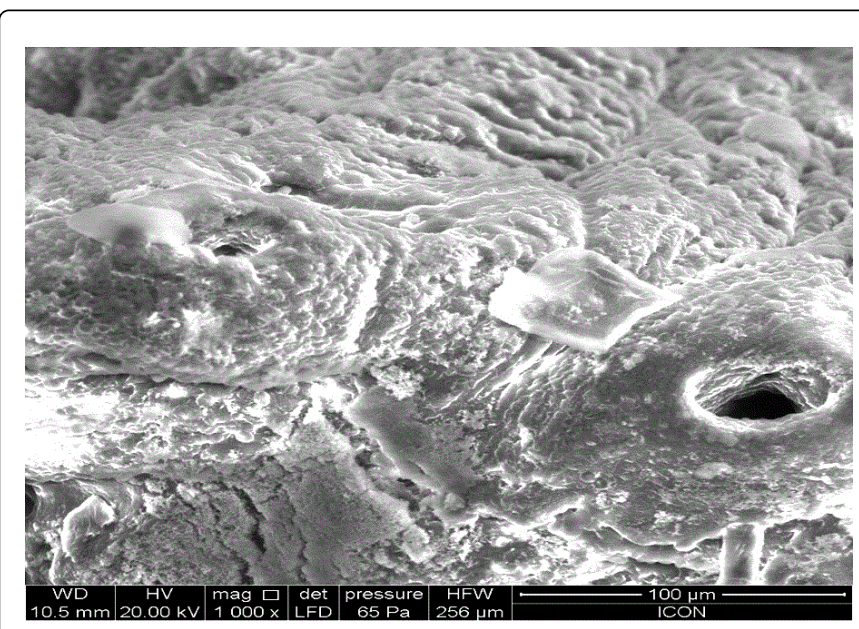

Figure 5: Regressed gland 1000X.

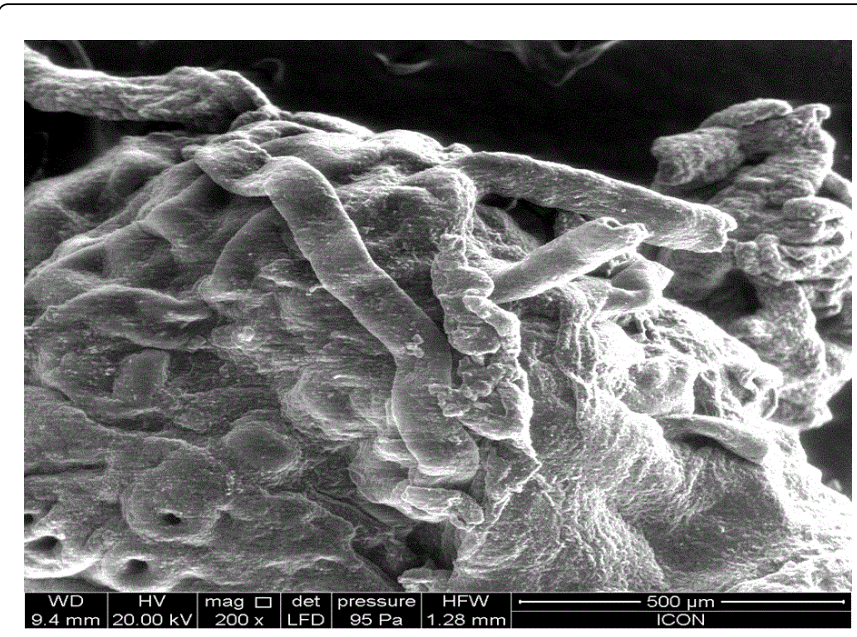

Figure 6: Developed vessels 200X.
The vessels also showed the phenomenon of angiogenesis evolving from day 2 to 5 (Figures 7-9) and then regressing on day 7 of progesterone (Figure 10) Observation of the sequential development of pinopodes glands and angiogenesis were considered as positive findings while absence of such development in any one component or all three components were considered as negative findings.

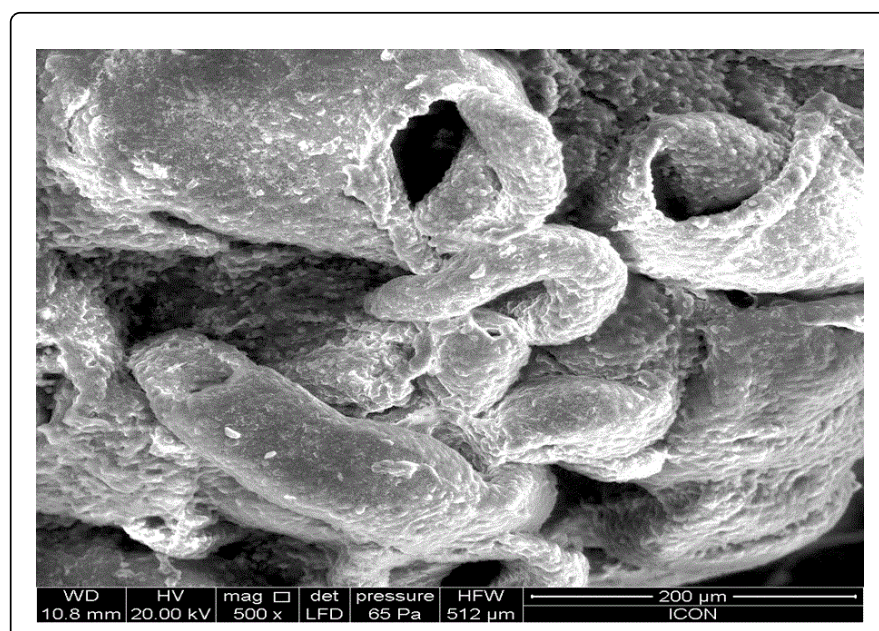

Figure 7: Developed vessel 500X.

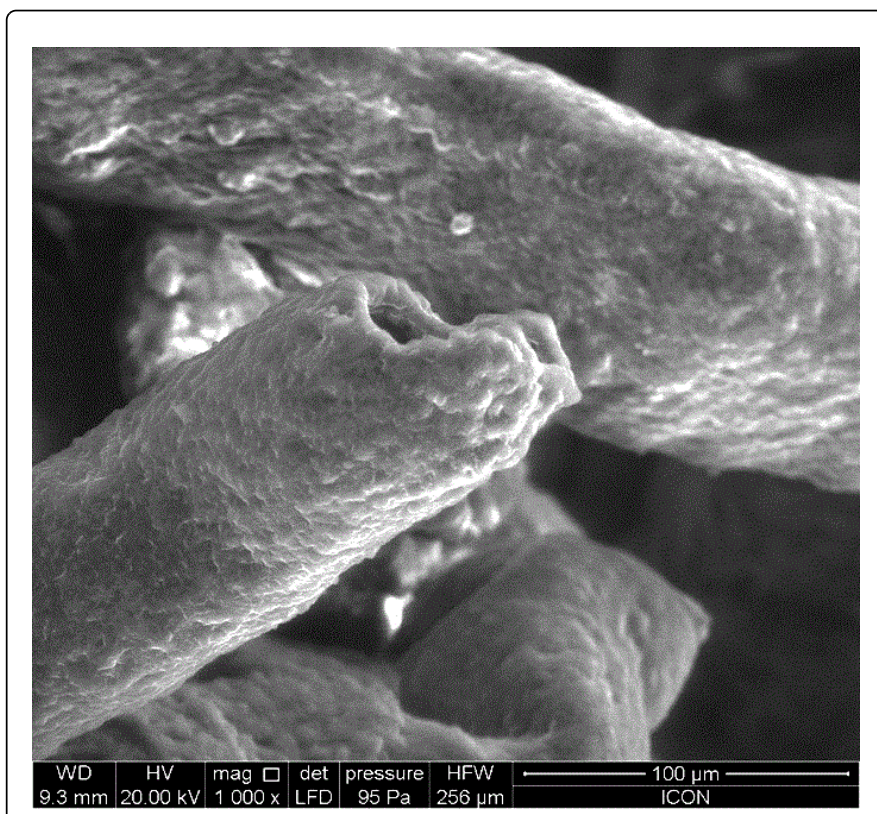

Figure 8: Developed vessels 1000X.

The observations in all the three components were consistently positively demonstrated in thirty eight patients. In two patients, the observations of pinopodes and glands were not convincing. 


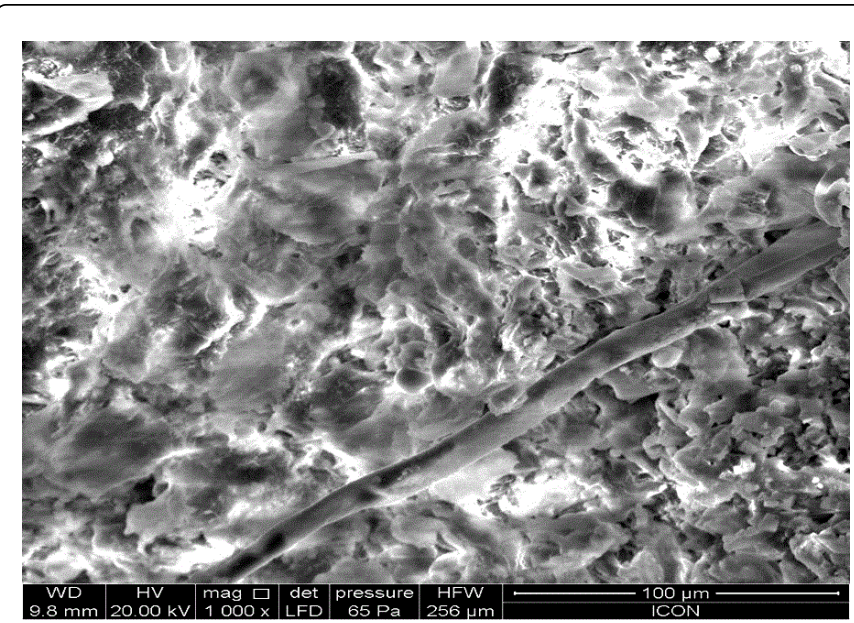

Figure 9: Regressed vessel 1000X.

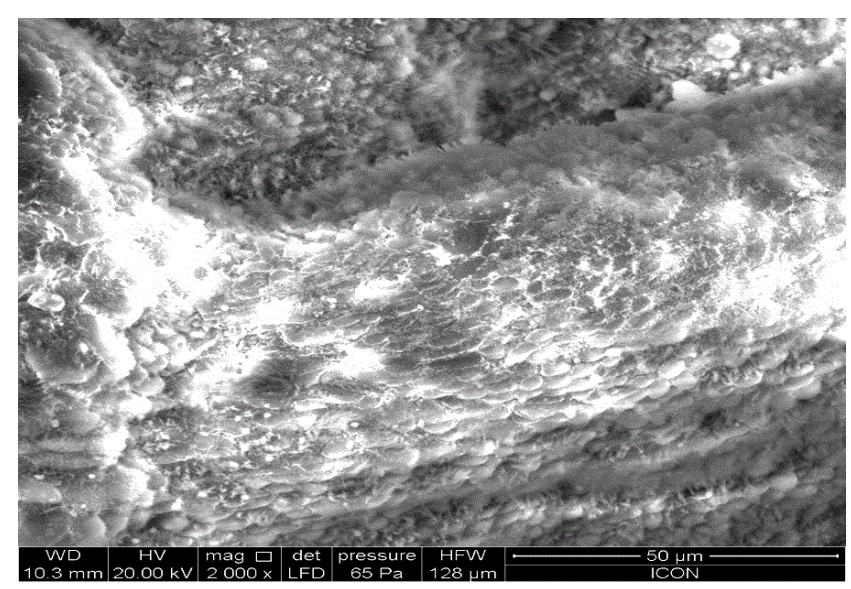

Figure 10: Regressing pinopodes 2000X.

\section{Discussion}

The endometriumis normally nonreceptive for the embryo, except during the window of implantation. Endometrial receptivity is a state when the endometrium allows the blastocyst to attach, penetrate \& finally invade the stroma. This process is called implantation. The synchronized development of the embryo to the stage of blastocyst and the differentiation of the endometrium to the receptive stage is necessary for the effective "cross-talk" which involves endocrine, paracrine and autocrine factors [5]. This short period is often referred to as "window of implantation" (WOI).

The endometrium undergoes a well-established series of histological changes under the influence of rising levels of estrogen and progesterone. The three components of the surface epithelium of the endometrium participate in the process of implantation. The luminal epithelium undergoes a change of formation of balloon like projections which are described as pinopodes. Pinopodes are considered as markers of endometrial receptivity in clinical practice [6]. In humans, pinopodes extend on the entire surface and cover the gland as well as vessels. In the present study, the fully developed pinopodes (FDP) were observed on fifth day of progesterone administration on already estrogen primed endometrium. The formation of the pinopodes on this day in the present study was so extensive that they were found to cover not only the surface of the glands but were even covering them throughout their entire depth. Interestingly, we could also see the vessels were also covered externally by pinopodes. They facilitate the adhesion of the blastocyst to the luminal epithelium by the mechanism of pinocytosis and endocytosis of uterine fluid [7].

Endometrial receptivity is heralded by the progesterone induced formation of pinopodes (also called uterodomes), which are surface epithelial cells that lose their microvilli and develop smooth protrusions appearing during the window of implantation. The pinopodes seem to absorb fluid from the uterine cavity forcing the blastocyst to be in contact with endometrial epithelium. Thus, the blastocyst adheres at the site of pinopodes. The most critical feature of the pinopodes is the removal of adhesion inhibiting.

Endometrial glands play very important role in the process of implantation. The glands change from proliferative to secretory during the window of implantation. The endometrial cells in the glands are rich in glycogen and lipids. The nourishment of human embryos is dependent on the contribution from the endometrial glands.

The third important endometrial component observed by scanning electron microscopy is the vessels. The phenomenon of the growth of the blood vessels from the pre-existing vessels is called as angiogenesis. Angiogenesis is the key feature of implantation. Researchers have shown three mechanisms of angiogenesis including sprouting, intussusception and elongation of the vessels in the endometrium $[8,9]$. In the present study, the process of angiogenesis is clearly observed as sprouting of vessels, along with their elongation and intussusception from the preexisting vessels. Interestingly, the higher magnifications show these branching vessels to be covered on their surface by pinopodes. The process of angiogenesis is induced by progesterone and is mediated through the growth factors. The growth factors which participate in the process of angiogenesis are angiopoeitins and vascular endothelial growth factor (VEGF). The angiopeitins Ang-1 and Ang-2 are upregulated during the window of implantation. They act in synergism with VEGF.

The search of predictors of implantation has focused on the analysis of various markers. A number of markers of receptive endometrium have been proposed which which include the members of integrin family $[10,11]$, glycodelin colony stimulating factor(CSF)and leukemia inhibiting factor(LIF) [12]. There are technologies capable of quantifying thousands of genes through DNA microarray [13] technologies. However, to date no single marker has been identified which is specific and sensitive in predicting the successful implantation.

As far as the histological changes in the luminal endometrial epithelium are concerned, previous workers have tried immunehistochemical assessment of the large number of endometrial proteins [14]. However, the results of such studies are controversial.

The advantage of studying the endometrium by electron microscopy over conventional histology is that of magnification of each endometrial component. In early 1950s, Noyes and coworkers examined the histological features of the endometrium by compound microscope and developed the technique of endometrial dating after the event of ovulation. The traditional method of dating endometrium enables both the morphology and function of the various endometrial components [14]. However, the criteria themselves were too variable to 
Citation: Patki SM, Patki SS, Patil RS, Patki US, Patil PS, et al. (2018) Electron Microscopy of Human Endometrium during Window of Implantation. J Cytol Histol 9: 516. doi:10.4172/2157-7099.1000516

Page 5 of 5

provide the accuracy to correctly assign the dating of the endometrium. Additionally, ovarian stimulation may lead to differences in the timing of endometrial maturation compound with the natural cycle. The variability of routine histological criteria was during the time of implantation. However, due to inter -observer subjectivity, it has limitations. On this background, the present study clearly shows three distinct markers of receptivity viz. appearance of pinopodes, angiogenesis and increase in number and diameters of glands.

The present study is focused on hormone replacement cycles for the detailed observation of the coordinated changes of the endometrial luminal surface components, which are consistently observed in all such patients. On the other hand, in stimulated cycles, supraphysiological steroid levels cause early closure of window of implantation due to uncoordinated response of endometrial components.

Similar are the observation in the study by Nikos and Collegues who demonstrated the formation of pinopodes during implantation window [15].

In the present study, fifth day of progesterone administration showed maximum changes of endometrial receptivity. Observation and documentation of such specific window of implantation in a cycle prior to the actual treatment cycle will help the clinician to do personalized embryo transfer in subsequent cycle. This will definitely improve the clinical pregnancy rate and successes of ART cycles.

\section{Conclusion}

The electron microscopic evaluation of the human luminal endometrium forms an important investigation in HRT cycles, especially prior to the procedure of actual embryo transfer. The three components of the endometrium viz. pinopodes, glands and vessels are observed to be showing evolving optimal changes which are necessary for the process of implantation from day 3 to day 5 of progesterone administration. Documentation of such positive changes can guide the clinician to identify the window of implantation and decide the day of embryo transfer accordingly. This will improve the implantation rates of ART procedures.

\section{References}

1. Boomsma CM, Macklon MS (2006) What can the clinician do improve implantation? Reprod Biomed Online 13: 845-855.

2. Acosta AA, Elberger L, Borghi M (2000) Endometrial dating and determination of window of implantation in healthy fertile women. FertilSteril 73: 788-798.

3. Paulson RJ, Sauer MV, Lobo RA (1990) Embryo implantation after human in vitro fertilization: Importance of endometrial receptivity. Fertil Steril 53: 870-874.

4. Edwards RG, Marcos S, Macnamee M et al(1991) High fecundity in amenorrhoic women in embryo transfer programmes. Lancet 338: 292-294.

5. Weterd of M, DeMayo F (2012) The progesterone receptor regulates implantation, decidualization and glandular development via a complex paracrine signaling network. Mol and cell endocrinology 357: 108-118.

6. George N (1999) Pinopodes as markers of endometrial receptivity in clinical practice. Human Reproduction 14: 99-106.

7. Bentin-Ley U (2000) Relevance of endometrial pinopodes for human blastocyst implantation. Hum Reprod 16: 67-73.

8. Koot Y, Teklenburg G, Salker M (2012) Molecular aspects of implantation failure. Biochimica et Biophysical Acta 1822: 1943-1950.

9. Gambino LS, WrefordNG, Bertram JF, Dockery P, Ledrmab F, et al. (2002) Angiogenesis occurs by vessel elongation in proliferative phase human endometrium. Human Reproduction 17: 1199-1206.

10. Lessey BA, Castlebasum AJ, Sawin SW (2000) Integrins as markers of uterine receptivity in women with primary unexplained infertility. Fertilsteril 63: 535-542.

11. Thomas K, Thomas A, Wood S (2003) Endometrial integrin expression in women undergoing in vitro fertilization and the association with subsequent outcome. FertilSteril 80: 502-507.

12. Ledee-Bataille N, Lapree-Delage G, Taupin JL (2002) Contration of leukemia inhibitory factor (LIF) in uterine flushing fluid is highly predictive of embryo implantation. Human Rproduction 17: 213-218.

13. Aplin J D (2006) Embryo implantation: the molecular mechanism remains elusive. Rprod Biomed online13: 833-839.

14. Cavagna M, Mantese JC (2003) Biomarkers of endometrial receptivity: A review. Placenta 24: 39-47.

15. Streus A, Nikas G, Sahil L, Eriksson H, Landgren B (2001) Formation of pinopodes in human endometrium is associated with the concentration of progesterone and progesterone receptors. FertilSteril 76: 782-791. 